\title{
Influence du pâturage et du gradient climatique sur la diversité floristique et la productivité des parcours naturels au Bénin
}

\author{
Yaya Idrissou ${ }^{1 *}$ Youssoufa Mama Sambo Seidou ${ }^{1}$ \\ Alassan Assani Seidou ${ }^{1}$ Hilaire Sorébou Sanni Worogo ${ }^{1}$ \\ Brice Gérard Comlan Assogba ${ }^{1}$ Ibrahim Alkoiret Traoré ${ }^{1}$ \\ Marcel Houinato ${ }^{2}$
}

\begin{abstract}
Mots-clés
Bovin, climat, pâturage, forêt protégée, Bénin
\end{abstract}

Submitted: 26 January 2019
Accepted: 5 March 2020
Published: 23 September 2020
DOI: $10.19182 /$ remvt.31894

\section{Résumé}

Située dans la zone soudanienne du Bénin, la forêt classée de l'Alibori supérieur est à cheval entre le climat tropical humide et le climat tropical sec. Elle est l'une des destinations privilégiées des transhumants nationaux et étrangers. Ces derniers sont attirés par ses ressources pastorales. Les fortes concentrations de bétail présentes ont des conséquences sur la biodiversité qu'il importe de connaître afin de prendre les décisions appropriées. Cette étude a eu pour objectif d'évaluer l'influence de l'intensité du pâturage et du gradient climatique sur la richesse et la diversité floristique, la phytomasse ainsi que sur la valeur pastorale de ces parcours. Les résultats ont montré que les effectifs des familles, genres et espèces diminuaient à mesure que l'intensité du pâturage augmentait. Ce même constat a été fait au niveau de la diversité maximale, de la phytomasse et de la capacité de charge. La zone soudanienne humide a montré une richesse et une diversité floristique, ainsi qu'une productivité et une capacité de charge meilleures que la zone soudanienne sèche. La valeur du recouvrement de la zone sèche a été plus élevée que celle de la zone humide, alors que sa valeur pastorale a été plus faible. Ces résultats pourraient contribuer à la gestion durable des parcours naturels des aires protégées et du système pastoral au Bénin.

- Comment citer cet article : Idrissou Y., Mama Sambo Seidou Y., Assani Seidou A., Sanni Worogo H.S., Assogba B.G.C., Alkoiret Traoré I., Houinato M., 2020. Influence of grazing and climatic gradient on the flora diversity and productivity of rangelands in Benin. Rev. Elev. Med. Vet. Pays Trop., 73 (3): 161-167, doi: $10.19182 /$ remvt.31894

\section{INTRODUCTION}

Les aires protégées sont des espaces voués à la conservation des ressources naturelles. Elles jouent un rôle capital dans la conservation de la biodiversité floristique et faunique (Marion, 2010). Aussi, représentent-elles un élément important d'aménagement du territoire car leur existence entraîne de nombreuses modifications dans les règles d'accès à l'espace (Triplet, 2009). Les aires protégées du Nord Bénin restent des destinations privilégiées pour les pasteurs sahéliens venant du Burkina Faso et du Niger (Lesse, 2016). Cela pourrait s'expliquer

\footnotetext{
1. Laboratoire d'écologie, santé et production animales (LESPA),

Faculté d'agronomie (FA), Université de Parakou (UP), 01 BP 123 Parakou, Bénin.

2. Laboratoire d'écologie appliquée (LEA), Faculté des sciences agronomiques

(FSA), Université d'Abomey-Calavi (UAC), Cotonou, Bénin.

* Auteur pour la correspondance

Tél. : + 22995521649 ; email : yayaidriss2617@gmail.com
}

par la présence d'abondantes ressources naturelles en comparaison avec la zone d'origine de ces pasteurs (Assani, 2017). Ainsi, la forêt classée de l'Alibori supérieur (FC-AS) du Nord Bénin est l'une des aires protégées fréquentées par les transhumants nationaux (Bénin) et étrangers (Niger, Burkina Faso, Nigeria, etc.) (Assani, 2017). Parmi les types de troupeaux fréquentant la forêt, Assani (2017) a identifié des transhumants transfrontaliers, communaux ou provenant de communes autres que les communes riveraines, et des transhumants installés à la lisière ou à l'intérieur de la forêt depuis plus d'un an. Plusieurs auteurs montrent que l'utilisation des aires protégées est depuis longtemps considérée comme une stratégie alternative par les éleveurs transhumants mais aussi sédentaires pour faire face à la saison sèche (Boutrais et al., 2008 ; Kaboré, 2010 ; Assani, 2017). L'utilisation de ces aires a des conséquences sur la biodiversité des parcours naturels.

Au Bénin, de nombreuses études portent sur la gestion durable des parcours naturels (Sinsin et Wotto, 2003 ; Djenontin, 2010 ; Lesse, 2016 ; Assani, 2017) à travers la réalisation d'enquêtes phytosociologiques. Cependant, très peu de chercheurs ont évalué l'influence du 
pâturage sur la diversité et la productivité des parcours naturels en général et celles des aires protégées en particulier. En effet, la productivité d'un pâturage est aussi influencée par des facteurs environnementaux comme la pluviométrie et la température. Ainsi, les travaux de Toko Imorou et al. (2010) relatifs à l'influence de la pluviosité sur la productivité des pâturages montrent que la quantité de pluie et sa répartition déterminent la variation de la biomasse herbacée.

Cette étude propose d'analyser l'influence du pâturage et du gradient climatique sur la biodiversité végétale et la productivité des parcours naturels de la FC-AS. Elle se justifie par le besoin de mettre en place une politique de gestion durable de la diversité biologique.

\section{MATERIEL ET METHODES}

\section{Milieu d'étude}

La forêt classée de l'Alibori supérieur au nord du Bénin a été créée par l'arrêté n 6459 du 20 août 1955. Elle couvre une superficie de 250205 hectares répartis sur plusieurs communes et sur les départements de l'Atacora (Kérou et Ouassa-Péhunco), du Borgou (Sinendé) et de l'Alibori (Banikoara, Kandi et Gogounou). Cette étude a été réalisée dans deux différentes zones climatiques de la FC-AS : la zone soudanienne sèche et la zone soudanienne humide (figure 1). La zone sèche est sous l'influence d'un climat tropical sec et comporte deux saisons : une saison pluvieuse allant de mai à mi-octobre et une saison sèche de mioctobre à avril (ASECNA, 2016). La pluviométrie annuelle moyenne est de $953 \mathrm{~mm}$ et la température annuelle moyenne est de $28,5^{\circ} \mathrm{C}$. Dans la zone humide, le climat est du type tropical subhumide avec deux saisons : une saison des pluies d'avril à fin octobre et une saison sèche de novembre à mars (ASECNA, 2016). La pluviométrie et la température annuelle moyenne sont respectivement de $1155 \mathrm{~mm}$ et de $26^{\circ} \mathrm{C}$.

\section{Dispositif expérimental}

Un dispositif aléatoire stratifié à deux facteurs et douze répétitions a été utilisé : a) zone climatique (zone soudanienne sèche et zone soudanienne humide), et b) intensité de pâturage (peu ou pas pâturé, moyennement pâturé et très pâturé). Pour choisir les parcours en fonction de l'intensité de pâturage au sein de chaque zone climatique, des enquêtes ont été réalisées auprès des éleveurs selon la méthode de Rakotoarimanana et al. (2008). Le parcours très pâturé se caractérisait par une fréquence de six heures de pâturage par jour de façon permanente durant toute l'année. Un effectif moyen de 25 bovins adultes et de cinq veaux pâturait sur un hectare de ce parcours. Ce parcours très pâturé était situé dans la zone tampon de la forêt classée où se trouvaient plusieurs sources d'eau temporaires. Il était accessible à tous les éleveurs. Le parcours moyennement pâturé était caractérisé par une fréquence de deux heures de pâturage par jour de façon permanente durant toute l'année. Un effectif moyen de 10 bovins adultes et de deux veaux pâturait sur un hectare de ce parcours. Il était situé dans le domaine classé de la forêt. L'exploitation de ce domaine avait été autorisée sous certaines conditions (s'acquitter de la contribution liée au pâturage, accepter de respecter les règles d'exploitation durable des ressources pastorales, être âgé d'au moins 18 ans, entre autres). Très peu d'éleveurs allaient dans ces zones car ils ne remplissaient pas les conditions prédéfinies. Le parcours peu ou pas pâturé était un domaine strictement interdit d'accès aux éleveurs par les agents des eaux et forêts. Il s'agissait du parcours situé dans le noyau central de la forêt.

Douze placettes de 100 mètres carrés chacune ont été positionnées aux quatre coins et au centre de chaque parcours (très pâturé, moyennement pâturé, et peu ou pas pâturé), de manière à cerner la variabilité spatiale des parcours. La richesse et la diversité floristique ont été évaluées sur les 12 placettes. La productivité, la valeur pastorale et la capacité de charge ont été estimées sur cinq placettes.

\section{Richesse et diversité floristique}

Pour chaque zone climatique, la richesse floristique (nombre de familles, de genres et d'espèces) a été évaluée sur les 12 relevés de chaque parcours (très, moyennement, peu ou pas pâturé). Les indices de diversité et de régularité floristiques estimés pour chaque parcours étaient les suivants :

- diversité maximale, $\operatorname{Hmax}=\log _{2} S$, où $S$ est le nombre total d'espèces dans chaque parcours ;

- indice de diversité de Shannon-Weaver, indice qui renseigne sur la diversité des espèces dans un milieu (Daget et Poissonet, 1971), et s'exprime par $H^{\prime}=-\sum_{i=1}^{i=n}(n i / N) \times \log _{2}(n i / N)$ où $N$ est le nombre total des espèces dans chaque parcours et $n i$ l'effectif de l'espèce $i$; - équitabilité ou équirépartition qui s'exprime par le rapport entre l'indice de diversité de Shannon-Weaver $(H$ ') et la diversité maximale (Hmax), soit $R=H^{\prime} / H \max$. Sa valeur varie de 0 à 1 . Une valeur qui tend vers 0 montre que la grande majorité des effectifs est concentrée sur une seule espèce, et une valeur qui tend vers 1 est la preuve que toutes les espèces ont la même abondance (Ramade, 1997) ;

- recouvrement global ; la méthode des relevés linéaires a été utilisée pour évaluer le recouvrement global des espèces. Deux lignes parallèles de $10 \mathrm{~m}$ chacune ont été positionnées au milieu de chaque placette (Daget et Poissonet, 1971). Sur chacune des lignes, des observations ont été effectuées tous les $20 \mathrm{~cm}$, ce qui donne un total de 100 observations $(\mathrm{N}=100)$ par placette. L'expression du recouvrement global $(R g)$ est $R g=(N-n i / N) \times 100$ où $n i$ représente le nombre d'observations du sol nu.

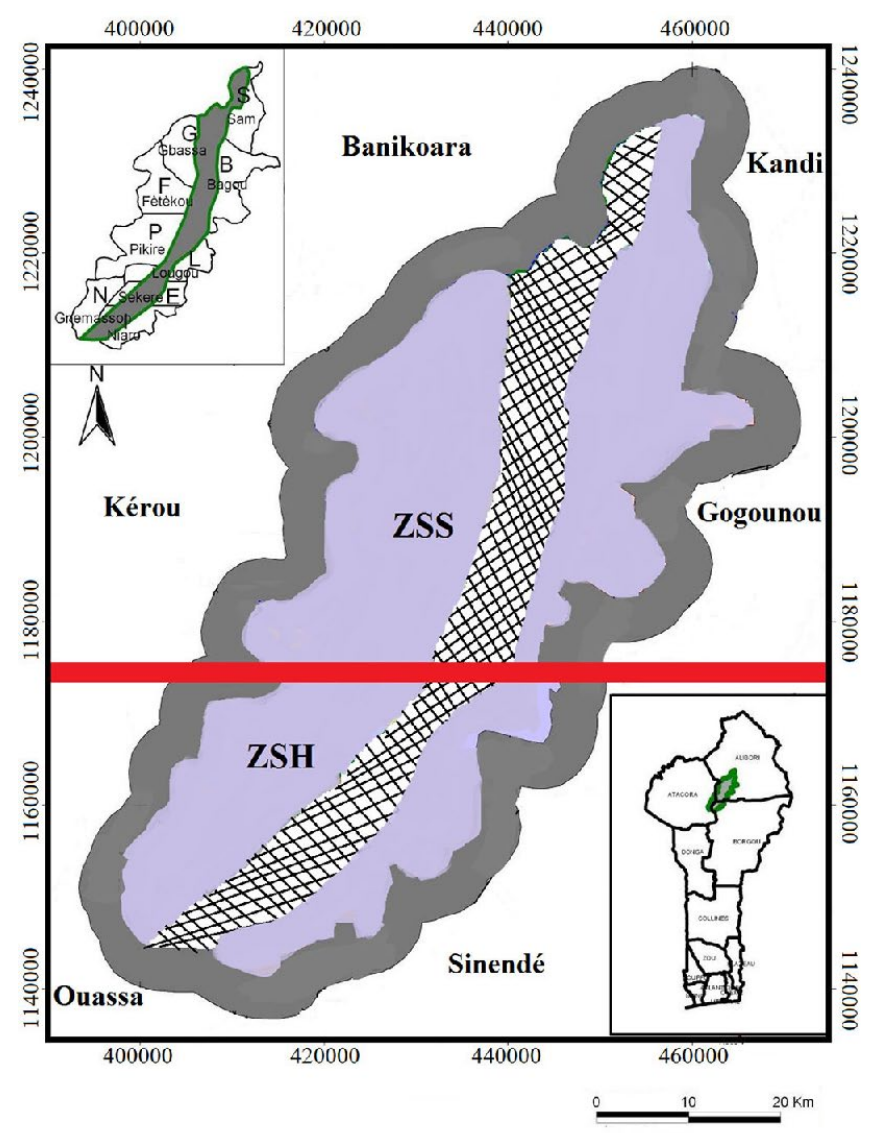

ZSS : Zone Soudanienne sèche : Domaine classé

ZSH : Zone Soudanienne Humide : Zone tampon Noyau central - Ligne de séparation des deux zones

Figure 1 : localisation de la zone d'étude au Bénin (adapté du Programme de gestion des forêts et terroirs riverains, 2016). 


\section{Productivité et capacité de charge}

Pour déterminer la productivité des différents parcours, cinq placettes de $100 \mathrm{~m}^{2}$ chacune par parcours (soit 15 placettes par zone climatique) ont été mises en défens au début de la saison pluvieuse jusqu'au pic de biomasse (mai à septembre). La méthode de la récolte intégrale a été utilisée pour déterminer la phytomasse au pic de biomasse (septembre 2017) sur des carrés de $1 \mathrm{~m}^{2}$ avec 30 répétitions par parcours placés de façon aléatoire à l'intérieur de chaque placette. Les échantillons de phytomasse collectés ont été pesés afin de déterminer le poids de la matière fraîche. Des échantillons de $100 \mathrm{~g}$ de matière fraîche par placeau ont été ensuite séchés à l'étuve à $105^{\circ} \mathrm{C}$ durant $48 \mathrm{~h}$, afin déterminer leur teneur en matière sèche. Les capacités de charge des parcours ont été estimées selon la méthode de Boudet (1991) : capacité de charge $=\frac{\text { phytomasse } \times 1000}{6,25 \times 3 \times 365}$ (avec la capacité de charge exprimée en UBT/ha/an et la phytomasse en t MS/ha).

\section{Valeur pastorale}

La valeur pastorale a été déterminée en attribuant à chaque espèce végétale un indice de qualité spécifique allant de 0 à 3 . Cette attribution a pris en compte les savoirs des éleveurs (Kiéma, 2007). Les espèces avec un indice spécifique (IS) égal à 0 sont sans valeur pastorale; les espèces à $\mathrm{IS}=1$ ont une faible valeur pastorale ; les espèces à IS $=2$ ont une valeur pastorale moyenne, et les espèces à IS $=3$ ont une bonne valeur pastorale. L'expression de la valeur pastorale est la suivante (Daget et

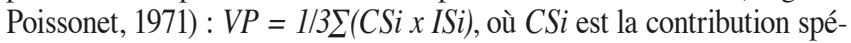
cifique de l'espèce $i$ et $I S i$ l'indice de qualité spécifique de l'espèce $i$.

\section{Analyses statistiques}

Pour étudier les effets de l'intensité du pâturage et du climat sur la diversité floristique et la productivité des parcours naturels, des modèles linéaires à effets mixtes (Bufford et Gaoue, 2015) ont été utilisés. Ces modèles ont été conçus à l'aide des packages lme4, nlme et glmmADMB (Fournier et al., 2012) dans le logiciel R, version 3.4.5 (R Core Team Development, 2018). Les modèles comprenaient l'intensité de pâturage, la zone climatique et l'interaction de ces deux facteurs comme effets fixes, et les placettes comme effets aléatoires. Les variables comme le nombre de familles, d'espèces et de genres ont été modélisées en utilisant une régression de Poisson. En revanche, les variables comme la diversité maximale, la diversité de Shannon-Weaver et l'équitabilité ont été modélisées en utilisant une régression bêta. Enfin, la productivité, la capacité de charge et la valeur pastorale ont été modélisées en utilisant une régression normale. Pour chaque variable de réponse, quatre modèles différents ont été testés et le modèle avec le plus bas critère d'information d'Akaïke (AIC) a été sélectionné (matériels supplémentaires I, II et III). Les résultats ont été présentés sous forme de graphiques réalisés à l'aide du package ggplot 2 du logiciel $\mathrm{R}$.

\section{RESULTATS}

\section{Richesse floristique}

Le nombre total d'espèces inventoriées sur l'ensemble des parcours était de 53. Ces espèces étaient réparties dans 40 genres et 20 familles dont les plus représentées étaient les Poaceae $(18,86 \%)$, les Leguminosae $(16,98 \%)$, les Fabacae (11,32 \%) et les Asteraceae $(9,43 \%)$ (tableau I).

\section{Tableau I}

Espèces inventoriées (53) sur les parcours de la forêt classée de l'Alibori supérieur au Bénin

\begin{tabular}{ll} 
Espèce & Famille \\
\hline Acalypha ciliata & Euphorbiaceae \\
Aeschynomene indica & Leguminosae \\
Ageratum conyzö̈des & Asteraceae \\
Alysicarpus ovalifolius & Papilionoideae \\
Andropogon fastigiatus Sw. & Poaceae \\
Andropogon gayanus Kunth & Poaceae \\
Andropogon pseudapricus & Poaceae \\
Brachiaria falsifiera (Trin.) Stapf & Poaceae \\
Brachiaria stigmatisata (Mez) Stapf & Poaceae \\
Cassia obtusifolia & Leguminosae \\
Crotalaria microcarpa & Papilionoideae \\
Crotalaria ochroleuca & Leguminosae \\
Desmodium gangeticum & Leguminosae \\
Desmodium velutinum & Fabaceae \\
Diospyros mespiliformis & Ebenaceae \\
Gmelina arborea & Lamiaceae \\
Hyptis suaveolens & Lamiaceae \\
Indigofera dendroides & Leguminosae \\
Indigofera hirsuta & Fabaceae \\
Indigofera leprieurii & Leguminosae \\
Leucas martinicensis & Lamiaceae \\
Lonchocarpus sericeus & Fabaceae \\
Ludwigia abyssinica A. Rich. & Onagraceae \\
Mangifera indica L. & Anacardiaceae \\
Melanthera abyssinica & Asteraceae \\
Melliniella micrantha Harms. & Leguminosae \\
&
\end{tabular}

\begin{tabular}{ll} 
Espèce & Famille \\
\hline Nelsonia canescens & Acanthaceae \\
Ocimum canum & Nepetoideae \\
Panicum pansum & Poaceae \\
Panicum phragmitoides Stapf & Poaceae \\
Paspalum scrobiculatum & Poaceae \\
Pennisetum pedicellatum (Trin.) & Poaceae \\
Pennisetum polystachion (L.) Schult. & Poaceae \\
Phyllantus muellerianus (Kuntze) Exell. & Euphorbiacea \\
Rhynchosia minima & Papilionoideae \\
Schwenckia americana & Solanaceae \\
Scoparia dulcis Linn. & Scrophulariaceae \\
Sesbania sudanica & Fabaceae. \\
Sida linifolia & Malvaceae \\
Sida urens & Malvaceae \\
Spermacoce radiata & Rubiaceae \\
Spermacoce stachydea & Rubiaceae \\
Synedrella nodiflora Gaertn. & Asteraceae \\
Tephrosia flexuosa & Leguminosae \\
Tephrosia pedicellata & Fabaceae \\
Tephrosia platycarpa Guill. et Perr. & Fabaceae \\
Tridax procumbens L. & Asteraceae \\
Triumphetta pentandra A. Rich. & Tiliaceae \\
Vicoa leptoclada & Asteraceae \\
Vigna racemosa & Leguminosae \\
Wahlenbergia perrottetii & Campanulaceae \\
Waltheria indica & Sterculiaceae \\
Wissadula amplissima & Malvaceae \\
&
\end{tabular}


La figure 2 présente l'effet du pâturage, du climat et de l'interaction de ces deux facteurs sur l'effectif de familles, genres et espèces des parcours naturels de la forêt. Le pâturage et le climat ont chacun eu un effet significatif $(p<0,001)$ sur le nombre de familles, de genres et d'espèces. L'interaction de ces deux facteurs n'a eu d'effet significatif ( $p<0,001$ ) que sur le nombre de familles (figure 2). Les effectifs de familles, genres et espèces ont été plus élevés dans le parcours peu pâturé que dans les parcours moyennement ou fortement pâturés (mat. suppl. IV). Par ailleurs, les effectifs ont été plus faibles dans la zone sèche que dans la zone humide.

\section{Diversité floristique}

Le pâturage a influencé significativement $(\mathrm{p}<0,05)$ la diversité maximale et l'équitabilité, mais il n'a eu aucun effet $(p>0,05)$ sur l'indice de diversité de Shannon-Weaver (figure 3). Le climat a eu une influence significative $(\mathrm{p}<0,001)$ uniquement sur la diversité maximale (figure 3 ). La valeur de la diversité maximale diminuait à mesure que l'intensité du pâturage augmentait. En revanche, la valeur de l'équitabilité a été plus élevée pour le parcours très pâturé que pour les parcours peu et moyennement pâturés (mat. suppl. V). La zone soudanienne sèche a eu une valeur de diversité maximale plus faible que la zone soudanienne humide. Cela signifiait que la flore herbacée de la zone humide était plus diversifiée que celle de la zone sèche. Toutefois, aucune différence significative ( $p>0,05)$ n'a été observée entre les valeurs d'équitabilité de ces deux zones. Par ailleurs, les espèces rencontrées dans la zone humide et sèche de la forêt classée étaient réparties de la même façon.

\section{Recouvrement et valeur pastorale}

L'effet du pâturage, du climat et de l'interaction de ces deux facteurs sur le recouvrement et la valeur pastorale des parcours naturels de la FC-AS sont résumés dans la figure 4. Le pâturage et le climat ont chacun eu un effet significatif $(\mathrm{p}<0,05)$ sur le recouvrement de la végétation herbacée épigée. L'interaction de ces deux facteurs a également un effet significatif $(\mathrm{p}<0,05)$ sur la valeur pastorale mais pas sur le recouvrement (figure 4). Le recouvrement et la valeur pastorale ont été plus faibles dans le parcours peu ou non pâturé que dans les parcours moyennement et très pâturés (mat. suppl. VI). La valeur du recouvrement de la végétation herbacée de la zone sèche a été plus élevée que celle de la zone humide. En revanche, la valeur pastorale de la zone humide a été plus élevée que celle de la zone sèche (mat. suppl. VI).

\section{Productivité et capacité de charge}

Le pâturage, le climat et l'interaction de ces deux facteurs ont influencé significativement $(\mathrm{p}<0,05)$ la productivité et la capacité de charge des parcours naturels de la FC-AS (figure 5). La productivité et la capacité de charge des parcours diminuaient à mesure que l'intensité du pâturage augmentait. La productivité et la capacité de charge des parcours de la zone sèche ont été faibles comparées à celles de la zone humide (mat. suppl. VII).

\section{DISCUSSION}

\section{Richesse floristique}

La végétation herbacée des parcours naturels de la forêt comprenait 53 espèces, reparties en 40 genres et 20 familles, nombres inférieurs à ceux obtenus par Gamoun et al. (2012) en Tunisie et par Assani (2017) dans la même zone d'étude que la nôtre. La différence entre nos résultats et ceux de Gamoun et al. (2012) pourrait s'expliquer par le fait que les deux études ne se sont pas déroulées dans la même zone. La différence avec les résultats d'Assani (2017) pourrait s'expliquer par le fait que la surface des relevés considérée par cet auteur est supérieure à la nôtre.

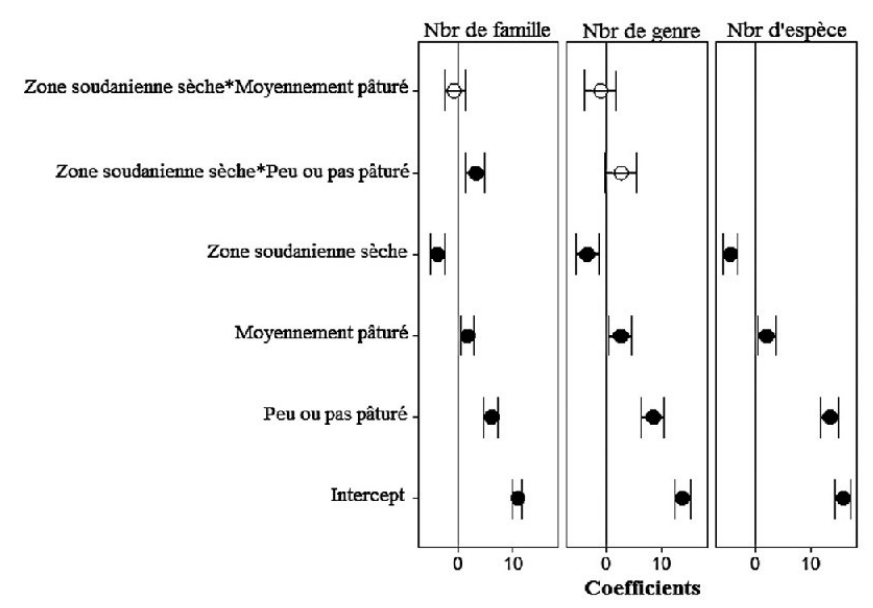

Figure 2 : effet du pâturage, du climat et de l'interaction des deux facteurs sur l'effectif de familles, genres et espèces des parcours naturels de la forêt classée de l'Alibori supérieur au Bénin. Les cercles noirs indiquent les variables significatives et les cercles blancs les variables non significatives.

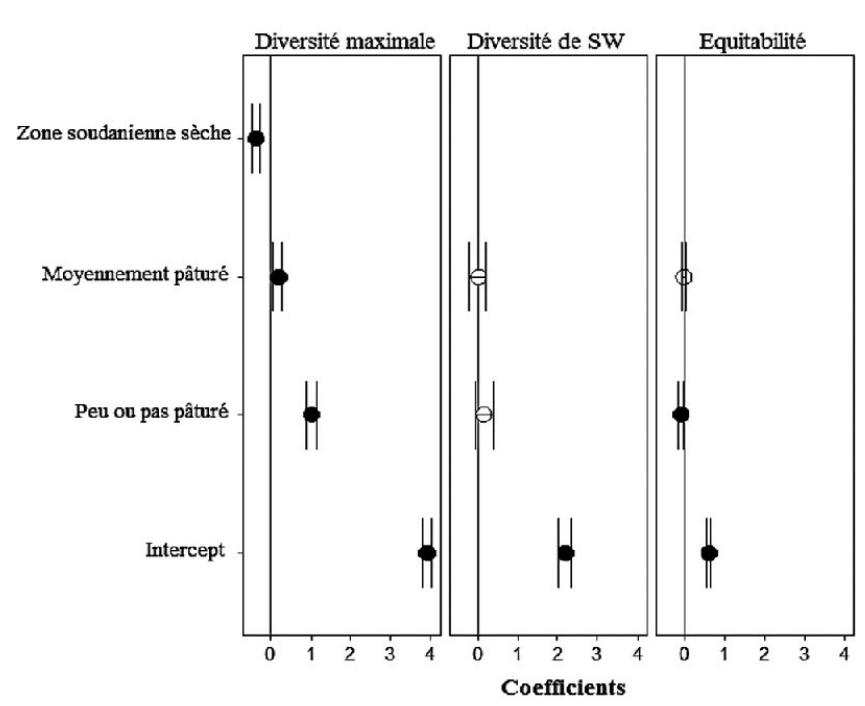

Figure 3 : effet du pâturage et du climat sur les indices de diversité floristiques des parcours naturels de la forêt classée de l'Alibori supérieur au Bénin. Les cercles noirs indiquent les variables significatives et les cercles blancs les variables non significatives.

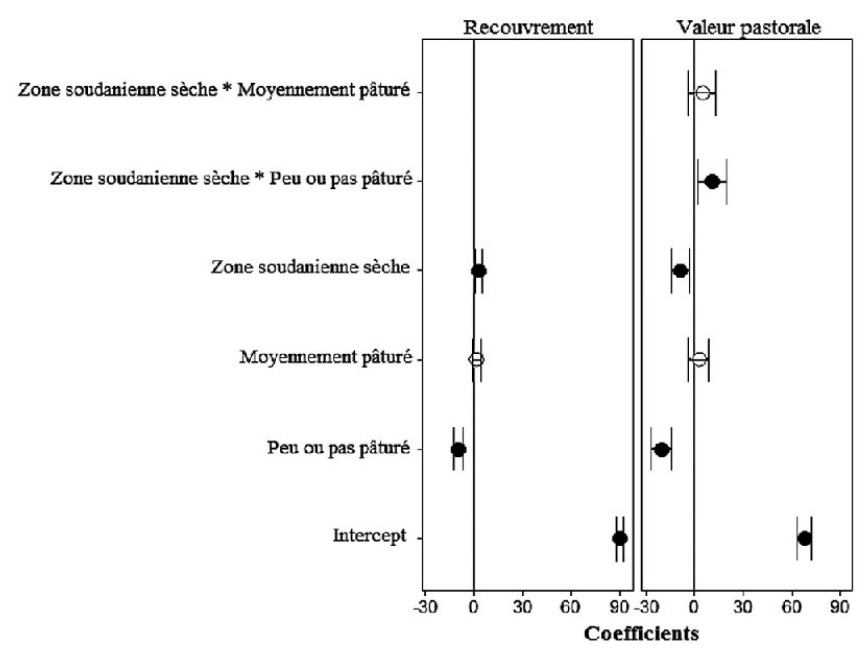

Figure 4 : effet du pâturage, du climat et de l'interaction des deux facteurs sur le recouvrement et la valeur pastorale des parcours naturels de la forêt classée de l'Alibori supérieur au Bénin. Les cercles noirs indiquent les variables significatives et les cercles blancs les variables non significatives. 


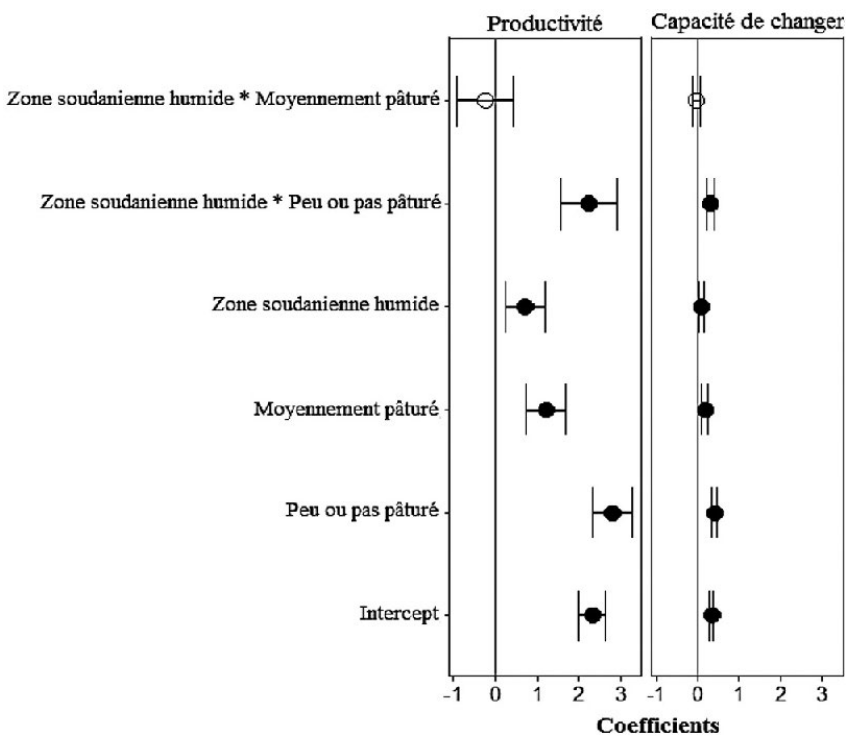

Figure 5 : effet du pâturage, du climat et de l'interaction des deux facteurs sur la productivité et la capacité de charge des parcours naturels de la forêt classée de l'Alibori supérieur au Bénin. Les cercles noirs indiquent les variables significatives et les cercles blancs les variables non significatives.

Concernant la composition floristique de la forêt, la famille des Poaceae a été la plus représentée, résultat qui corrobore celui d’Assani (2017) dans la même zone. La forte proportion de Poaceae pourrait s'expliquer par le fait que cette famille possède une forte vitesse de repousse et une forte possibilité de tallage (Kouassi et al., 2014). Par ailleurs, les Poaceae ont une forte résistance aux conditions climatiques difficiles et sont atteintes rarement de maladies.

Les effectifs de familles, de genres et d'espèces diminuaient à mesure que l'intensité du pâturage augmentait. Des résultats similaires ont été rapportés par Akpo et Grouzis (2000) ; une forte intensité de pâturage entraîne une diminution de la richesse floristique des parcours, tout en permettant aux Poaceae et aux xérophytes de s'installer. En revanche, nos résultats sont contraires à ceux d'autres auteurs (Boutrais, 1994 ; Hatfield et al., 2006) qui rapportent que le pâturage enrichit les formations herbacées des parcours. Cet effet du pâturage sur les formations végétales n'est valable que si la capacité de charge du pâturage est respectée, sinon s'ensuit une dégradation progressive du pâturage.

Les effectifs de familles, de genres et d'espèces des différents parcours de la zone soudanienne humide ont été plus élevés que ceux de la zone soudanienne sèche. Cela pourrait s'expliquer par la différence de climat dans les deux zones. Le pâturage n'affecte pas à lui seul directement la variabilité de la richesse floristique qui est beaucoup plus influencée par la pluviométrie (Westbrooke et al., 2005) ; la pluviométrie enregistrée dans la zone humide est plus élevée que celle observée dans la zone sèche (ASECNA, 2016).

\section{Diversité floristique}

La diversité d'une formation végétale est appréciée grâce aux indices de diversité floristique (Ramade, 1997). La présente étude a révélé que l'intensité de pâturage réduit significativement l'indice de diversité maximale. Le pâturage a ainsi provoqué dans la présente étude une inégalité des contributions individuelles des espèces. Ces résultats sont semblables à ceux obtenus par Rakotoarimanana et al. (2008) dans une savane des Hautes Terres malgaches et par Hiernaux (1998) au Sahel. Ces auteurs montrent que la dominance d'un petit nombre d'espèces s'établit à mesure que l'intensité de pâture augmente. En revanche, ils s'opposent à ceux rapportés par Rakotoarimanana et Grouzis (2006). Selon Balent et al. (1998), un pâturage moyen entraîne une augmentation de la diversité spécifique des prairies, alors qu'un pâturage intense provoque sa diminution.

La grande diversité de la flore des différents parcours de la zone humide comparée à la flore des parcours de la zone sèche aurait une origine climatique ; la zone humide située dans le département du Borgou reçoit plus de pluie (900 à $1400 \mathrm{~mm} / \mathrm{an}$ ) que la zone sèche (700 à $1200 \mathrm{~mm} / \mathrm{an}$ ) située dans le département de l'Alibori. Ce résultat est en accord avec les travaux de N'da et al. (2008).

\section{Recouvrement et valeur pastorale}

L'intensité du pâturage a augmenté le recouvrement de la végétation herbacée épigée. On peut en déduire que le pâturage a un effet stimulant sur les plantes (Daget et Godron, 1995). L'appréciation de la qualité d'un parcours dépend non seulement de la teneur en éléments nutritifs et de la digestibilité des fourrages qu'il contient, mais aussi de sa valeur pastorale. Cet élément est primordial dans l'appréciation de la qualité d'un parcours. Nos résultats ont montré que la valeur pastorale des parcours augmentait à mesure que le pâturage s'intensifiait. Ce résultat est lié au rapport entre plantes à indice de qualité élevé et plantes à indice nul. Des résultats similaires ont été rapportés par Rakotoarimanana et al. (2008).

\section{Productivité et capacité de charge}

Les résultats ont révélé que la productivité et la capacité de charge des parcours diminuaient lorsque le pâturage s'intensifiait. Ceci était attendu puisque la phytomasse totale sur pied était plus élevée sur une zone non pâturée que sur une zone pâturée. Ces résultats corroborent ceux rapportés par d'autres auteurs (Rakotoarimanana et al., 2008 ; Altesor et al., 2005). La productivité et la capacité de charge obtenues dans la zone humide ont été supérieures à celles de la zone sèche. Ces résultats sont dus au climat qui règne dans chaque zone. Pour Rivière (1978) la productivité d'un pâturage est principalement fonction de la zone climatique et à l'intérieur d'une même zone climatique on peut noter des variations importantes selon la nature des sols et le type d'aménagement en place.

\section{CONCLUSION}

Les résultats ont révélé que le pâturage avait une influence sur la diversité floristique et la productivité des parcours. A mesure que le pâturage s'intensifiait, on assistait à une diminution de la richesse spécifique, de la diversité floristique, de la productivité et de la capacité de charge des parcours. En revanche, le pâturage intense améliorait la qualité fourragère et le recouvrement de la végétation herbacée. Ces résultats pourraient contribuer à mettre en place une politique de gestion durable de la diversité biologique des parcours naturels de la FC-AS. Il serait ainsi opportun que cette étude soit étendue aux autres parcours naturels du pays.

\section{Remerciements}

Ce travail a été financé par la Fondation internationale pour la science (IFS) à travers la subvention D/5824-1 accordée à A.A. Seidou. Les auteurs expriment leur reconnaissance à cette fondation.

\section{Déclaration des contributions des auteurs}

YI, AAS et BGCA ont participé à la conception et à la planification de l'étude. YMSS et HSSW ont collecté et saisi les données de terrain. YI et AAS ont réalisé les analyses statistiques, interprété et rédigé la première version du manuscrit. IAT et $\mathrm{MH}$ ont participé à la révision 
critique du manuscrit et ont donné leur accord sur la version finale à publier. Tous les auteurs autorisent la publication de la version finale.

\section{Conflits d'intérêts}

L'étude a été réalisée sans aucun conflit d'intérêts.

\section{REFERENCES}

Akpo L.E., Grouzis M., 2000. Valeur pastorale des herbages en région soudanienne: le cas des parcours sahéliens du Nord-Sénégal. Tropicultura., 18 (1): 1-8

Altesor A., Oesterheld M., Leoni E., Lezama F., Rodríguez C., 2005. Effect of grazing on community structure and productivity of a Uruguayan grassland. Plant Ecol., 179: 83-91, doi: 10.1007/s11258-004-5800-5

ASECNA, 2016. Données climatiques de la zone Nord-Station de Kandi et de Parakou République du Bénin

Assani S.A., 2017. Transhumance dans la forêt classe de l'Alibori Supérieur au nord du Bénin: acteurs, pratiques d'élevage et modèle conceptuel de I'exploitation des ressources pastorales. Thèse Doct., Université de Parakou, Bénin, 201p.

Balent G., Alard D., Blanfort V., Gibon A., 1998. Activités de pâturage, paysages et biodiversité. Ann. Zootech., 47: 419-29, doi: 10.1051/animres:19980509

Boudet G., 1991. Pâturages Tropicaux et Cultures Fourragères. Coll. Manuels et Précis d'Elevage n4, IEMVT. Minist. Coop., France, 266 p.

Boutrais J., 1994. Les Foulbé de l'Adamaoua et l'élevage: de l'idéologie pastorale à la pluri-activité. Cah. Etudes Afr., 34 (133-135): 175-196, doi: 10.3406/cea.1994.2046

Boutrais J., Aubertin C., Rodary E., 2008. Pastoralisme et aires protégées d'Afrique de l'Ouest en regard de l'Afrique de I'Est. In: Aires Protégées Espac. Durables? IRD Editions, Marseille, France, 215-246, doi: 10.4000/ books. irdeditions. 5687

Breman H., Ridder N., 1991. Manuel sur les pâturages des pays sahéliens. Ed. Karthala, ACCT, ABOL-DLO et CTA, 485 p.

Bufford J.L., Gaoue O.G., 2015. Defoliation by pastoralists affects savanna tree seedling dynamics by limiting the facilitative role of canopy cover. Ecol. Appl. 25 (5): 1319-1329, doi: 10.1890/14-0953.1

Daget P., Godron M., 1995. Troupeaux, espaces et sociétés. Collection «Universités francophones», Hatier, Aupelf, Uref, Evreux, France, 510 p.

Daget P., Poissonet J., 1971. Une méthode d'analyse phytologique des prairies. Ann Agron., 22 (1): 5-41

Djenontin J., 2010. Dynamique des stratégies et des pratiques d'utilisation des parcours naturels pour l'alimentation des troupeaux bovins au NordEst du Bénin. Thèse Doct., Université d'Abomey-Calavi, Bénin, 274 p.

Fournier D.A., Skaug H.J., Ancheta J., lanelli J., Magnusson A., Maunder M.N., Nielsen A., et al., 2012. AD Model Builder: using automatic differentiation for statistical inference of highly parameterized complex nonlinear models. Optim. Methods Softw. 27 (2): 233-249, doi: 10.1080/10556788.2011.597854

Gamoun M., Ouled Belgacem A., Hanchi B., Neffati M., Gillet F., 2012. Effet du pâturage sur la diversité floristique des parcours arides du sud Tunisien. Rev. Ecol. Terre Vie, 67 : 271-282
Hatfield R., Davies J., Wane A., Kerven C., Dutilly-Diane C., Biber J.P., Merega J.L., et al. 2006. Revue mondiale de l'économie du pastoralisme. L'Initiative Mondiale pour un Pastoralisme Durable, IUCN, Nairobi, Kenya, $51 \mathrm{p}$.

Hiernaux P., 1998. Effects of grazing on plant species composition and spatial distribution in rangelands of the Sahel. Plant Ecol., 138: 191-202, doi: 10.1023/A:1009752606688

Kaboré A., 2010. Brousse des uns, aire protégée des autres, histoire du peuplement, perceptions de la nature et politique des aires protégées dans le Gourma burkinabè: I'exemple de la Réserve partielle de faune de Pama. Thèse Doct., Institut de Hautes Etudes Internationales et du Développement, Genève, Suisse, 383 p.

Kiéma S., 2007. Elevage extensif et conservation de la diversité biologique dans les aires protégées de l'Ouest Burkinabé : arrêt sur leur histoire, épreuves de la gestion actuelle, état et dynamique de la végétation. Thèse Doct. Université d'Orléans, France, 707 p.

Kouassi A.F., Koffi, K.J., N'Goran, K.S.B., Ipou, I.J., 2014. Potentiel de production fourragère d'une zone pâturée menacée de destruction: cas du cordon littoral Port-Bouët et Grand-Bassam. J. Appl. Biosci., 82: 74037410, doi: 10.4314/jab.v82i1.13

Lesse D.P., 2016. Gestion et modélisation de la dynamique des parcours de transhumance dans un contexte de variabilités climatiques au nord-est du Bénin. Thèse Doct., Université d'Abomey-Calavi, Bénin, 299 p.

Marion B., 2010. Impact du pâturage sur la structure de la végétation : interactions biotiques, traits et conséquences fonctionnelles. Thèse Doct., Université de Rennes, France, 235 p.

N'da D., Adou Y.C.Y., N'guessan K.E., Kone M., Sagne Y.C., 2008. Analyse de la diversité floristique du parc national de la Marahoué, Centre-Ouest de la Côte d'Ivoire. Afr. Sci. Rev. Int. Sci. Technol., 4(3): 552 - 579, doi: 10.4314/afsci.v4i3.61700

R Core Team Development, 2018. R: A language and environment for statistical computing. R Foundation for Statistical Computing, Vienna, Austria. 2012. URL Http.www R-Proj. Org.

Rakotoarimanana V., Gondard H., Ranaivoarivelo N., Carriere S., 2008. Influence du pâturage sur la diversité floristique, la production et la qualité fourragères d'une savane des Hautes Terres malgaches (région de Fianarantsoa). Sécheresse, 19: 39-46

Rakotoarimanana V., Grouzis M., 2008. Short Term Effects of Burning and Grazing on the Forage Quality of a Heteropogon contortus- Dominated Savanna in the Southwest of Madagascar. Rev. Elev. Med. Vet. Pays. Trop., 61 (2): 81-88, doi: 10.19182/remvt.10003

Ramade F., 1997. Conservation des écosystèmes méditerranéens. Les fascicules du plan bleu, Economica, Paris, France, 189 p.

Rivière R., 1978. Manuel d'alimentation des ruminants domestiques en milieu tropical. I.E.M.V.T, République de France, Ministère de la Coopération, $527 \mathrm{p}$.

Sinsin B., Wotto J., 2003. Changes in floristic composition of grazing land in northern Sudanian zone (Benin), In: Rangelands in the New Millennium, VIlth International Rangeland Congress, Durban, South Africa, 402-404

Toko Imorou I., Arouna O., Sinsin B., 2010. Cartographie des changements spatio-temporels de l'occupation du sol de la forêt classée de l'Alibori Supérieur au Nord-Bénin. Ben. Géo., 7: 22-39

Triplet P., 2009. Manuel de gestion des aires protégées d'Afrique francophone. Awely, Paris, France, 1251 p.

Westbrooke M.E., Florentine S.K., Milberg P., 2005. Arid land vegetation dynamics after a rare flooding event: influence of fire and grazing. J. Arid Environ., 61 (2): 249-260, doi: 10.1016/j.jaridenv.2004.09.004 


\section{Summary}

Idrissou Y., Mama Sambo Seidou Y., Assani Seidou A., Sanni Worogo H.S., Assogba B.G.C., Alkoiret Traoré I., Houinato M. Influence of grazing and climatic gradient on the floristic diversity and productivity of rangelands in Benin

Located in the Sudanian zone of Benin, the Upper Alibori classified forest straddles the humid tropical and the dry tropical climates. It is one of the privileged destinations of national and foreign transhumant herders. The latter are attracted by its pastoral resources. The high concentration of livestock there has consequences on the biodiversity that should be known in order to make adequate decisions. The objective of this study was to assess the influence of grazing intensity and climatic gradient on the floristic richness and diversity, phytomass as well as on the pastoral value of these rangelands. The results showed that the numbers of families, genera and species decreased as the intensity of grazing increased. The same observation was made at the level of maximum diversity, phytomass and carrying capacity. The wet Sudanian zone showed better floristic richness and diversity, as well as better productivity and carrying capacity than the dry Sudanian zone. The cover value of the dry zone was higher than that of the wet zone, whereas its pastoral value was lower. These results could contribute to the sustainable management of rangelands in protected areas and of the pastoral system in Benin.

Keywords: cattle, climate, grazing, protected forests, Benin

\section{Resumen}

Idrissou Y., Mama Sambo Seidou Y., Assani Seidou A., Sanni Worogo H.S., Assogba B.G.C., Alkoiret Traoré I., Houinato M. Influencia del pastoreo y del gradiente climático sobre la diversidad florística y la productividad de los recorridos naturales en Benín

Situada en la zona sudanesa de Benín, el bosque protegido de Alibori superior se encuentra a caballo entre el clima tropical húmedo y el clima tropical seco. Es uno de los destinos privilegiados de los trashumantes nacionales y extranjeros. Estos últimos son atraídos por sus recursos pastorales. Las fuertes concentraciones de ganado presentes tienen consecuencias sobre la biodiversidad, las cuales deben conocerse con el fin de tomar decisiones apropiadas. El objetivo de este estudio fue evaluar la influencia de la intensidad de pastoreo y su gradiente climático sobre la riqueza y la biodiversidad florística, la fitomasa, así como sobre el valor pastoral de los recorridos. Los resultados mostraron que la cantidad de familias, géneros y especies disminuyeron conforme la intensidad de pastoreo aumentó. Este mismo constado se observó a nivel de la diversidad máxima, de la fitomasa y de la capacidad de carga. La zona sudanesa húmeda mostró una riqueza y una diversidad florística, así como una productividad y capacidad de carga superiores que la zona sudanesa seca. El valor de la cobertura de la zona seca fue más elevado que el de la zona húmeda, mientras que su valor pastoral fue más bajo. Estos resultados podrían contribuir a la gestión sostenible de los trayectos naturales en las áreas protegidas y del sistema de pastoreo en Benín.

Palabras clave: ganado bovino, clima, pastoreo, monte protegido, Benin 
